\title{
A brief overview of the pathogenesis of scleroderma (systemic sclerosis)
}

\author{
E Carwile LeRoy
}

Although definitive answers are not yet forthcoming, understanding the mechanisms of fibrosis in scleroderma might improve the relative therapeutic vacuum now surrounding not only generalised scleroderma (systemic sclerosis (SSc)) but also the fibrosis of keloid formation, cirrhosis of the liver, pulmonary fibrosis, and atherosclerosis as well. Discussion here will include immune, vascular, and fibroblast/ extracellular matrix features of SSc. ${ }^{1}$

\section{Immune features}

Systemic sclerosis has been viewed as autoimmune since the introduction of indirect immunofluorescent antinuclear antibody determinations in the 1950s. Before the routine use (in the 1980s) of rapidly dividing, human cell substrates (HEp-2), SSc serum samples were only infrequently antinuclear antibody positive; since the introduction of $\mathrm{HEp}-2$ cells more than $90 \%$ of serum samples from patients with SSc are antinuclear antibody positive. The table lists the various reactivities. Most of these are reported as either speckled or nucleolar pattern. The anticentromere antibody pattern is distinctive and, together with nailfold capillary examinations, has added considerably to the detection of the patient prone to connective tissue disease who presents with Raynaud's phenomenon. Patients with anticentromere antibodies, with only rare exceptions, develop over the years limited cutaneous SSc. Most other antinuclear antibody specificities associated with SSc require subsidiary testing for precise assignment and, although some seem to be associated with selected HLA haplotypes, clinical and prognostic associations are not yet definite. ${ }^{2}$ Immunoblotting and enzyme linked immunosorbent assay (ELISA) techniques for antinuclear antibody determination are under study; unfortunately, these sensitive procedures may generate unnecessary (false positive) referrals.

Autoimmune serology in systemic sclerosis $(S S c)$

Anticentromere antibodies

Antinuclear antibodies (speckled, nucleolar, other) Antinucleolar antibodies

RNA polymerase I

Fibrillarin (U3 RNA protein complex)

Nucleolar 4-65 RNA

U2 RNA protein complex

Polymyositis/SSc overlap $(\mathrm{Pm} / \mathrm{Scl})$

Antitopoisomerase I (formerly Scl-70)

Antitopoisomerase I (formerly Scl-70)

Anticollagen type IV (basement membrane structure)

Antilaminin (basement memb

Jo-1

SS-A(Ro), SS-B(La)
Emerging data from several laboratories indicate that a network of $T$ lymphocytes is overactive in SSc. ${ }^{3}$ It is well known that most of the cutaneous cellular (perivascular and interstitial) infiltrates in SSc are $\mathrm{T}$ cells. Only recently has the $T$ cell hyperactivity been localised to the T helper, CD4 positive, interleukin 2 dependent arm of the immune response. ${ }^{4}$ Circulating SSc T cells can be shown to be reactive to the basement membrane component laminin, ${ }^{5}$ to certain retroviral proteins ${ }^{6}$ and, recently, to type I collagen ${ }^{7}$; in addition, enhanced lymphocyte adhesion to endothelial cells can also be shown. ${ }^{8}$ This raises the intriguing possibility that $\mathrm{T}$ cells are autoreactive to the endothelium (endothelial cell membrane, basal lamina matrix, others) throughout the vascular system. The precise autoepitopes of this reactivity can now be identified.

There are a variety of potential mechanisms by which T cells might become activated in SSc. They could be predisposed by the expression of a class II major histocompatibility complex gene on autologous antigen presenting cells to react strongly with any of the components listed as autoantigenic epitopes (table). Were these epitopes integral structural components of the vasculature, a $\mathrm{T}$ cell directed attack on the vascular system could be self perpetuating. This hypothesis would predict that the $\mathrm{T}$ cell hyperactivity in SSc to vascular structures would be mediated through the antigen specific $\mathrm{T}$ cell receptor (CD3)-in effect, the classic autoimmune hypothesis.

There are other ways to activate $T$ cells in SSc. The increased expression of extracellular matrix components by SSc fibroblasts, as well as the major alterations in the structure and function of the endothelium, creates tissue microenvironments likely to exhibit extracellular matrix epitopes which interact with $\mathrm{T}$ lymphocytes through a family of attachment proteins known as beta one ( $\beta 1)$ integrins or VLA (very late antigen) $T$ cell membrane molecules. ${ }^{9}$ Binding of integrins by the matrix stimulates T cells by mechanisms termed costimulation. ${ }^{10}$

At least four matrix molecules may costimulate $T$ cells already engaged by antigen specific mechanisms: $(a)$ collagen (especially the basal lamina type IV molecule with many non-helical and therefore immunogenic epitopes) ${ }^{11} ;(b)$ fibronectin (especially 'cellular' fibronectin expressing the type III connecting segment termed the CS-1 sequence $)^{12} ;(c)$ laminin and its analogue merosin ${ }^{13}$; and $(d)$ the multimeric fibronectin-like molecule cytotactin(tanascin). ${ }^{14}$ 
Costimulation of $\mathrm{T}$ cells by matrix molecules is an intriguing phenomenon just beginning to be understood. In vitro engagement of the $T$ cell receptor is a necessary but insufficient stimulus for $T$ cell propagation; in fact, $T$ cell receptor engagement in the absence of costimulatory signals makes the $T$ cell tolerant rather than stimulating it; it is only in the presence of costimulatory signals that full $\mathrm{T}$ cell amplification occurs. In SSc, as in most other autoimmune disorders, the major $\mathrm{T}$ cell amplification is that of a subset consisting of CD4 positive, $\mathrm{T}$ helper, interleukin 2 dependent cells. Curiously, when the $T$ cell receptor is engaged-for example, non-specifically by a monoclonal antibody (to CD3)-receptors for costimulation (VLAs) are not changed in number but are rendered more able (energised, activated) to induce mitogenesis, interleukin 2 secretion, and $T$ cell amplification. ${ }^{15}$

Fibronectin, a multidomain attachment protein, binds to $\mathrm{T}$ cells through two receptors, VLA-4 and VLA-5; VLA-4 recognises in fibronectin a 10 amino acid sequence in the highly variable, alternatively spliced region of the molecule termed IIICS or CSl. This sequence, gly - pro - glu - iso - leu - asp - val - pro - ser - thr (GPEILDVPST), with its rigid prolines and charged constituents, might be expected to bind avidly (and additionally to be highly immunogenic). This domain is sparse in plasma but plentiful in 'cellular' fibronectin. Whereas $T$ cells and natural killer cells bind fibronectin by both VLA-4 and VLA-5, B cells, capable of presenting antigen to $T$ cells, bind fibronectin only through VLA-4. Returning to $\mathrm{T}$ cells, another site of fibronectin binding is between the classic arg-gly-asp (RGD) cell binding ligand and VLA-5. Such intricacy provides ample opportunity for cooperation between costimulatory receptors, and additive, synergistic, or other effects of signal transduction between these richly multiepitope ligands and such amply decorated $\mathrm{T}$ cell membranes. Little wonder that the quantitative effects of costimulation by matrix molecules are so impressive (in vitro). ${ }^{16}$

Laminin, the major constituent of the lamina rara of basal lamina of epithelia and endothelia, is a trimolecular complex resembling, when assembled, the Coptic Christian cross. Laminin which in other cell types shows binding affinity for VLA-1, VLA-2, VLA-3, and VLA-6, binds T cells only through VLA-6. Full mapping of the epitope specificity of laminin-VLA-6 interaction on $T$ cells awaits future study. ${ }^{17}$

Collagens were among the first extracellular molecules shown to bind several integrins (VLA-1, VLA-2, VLA-3) in a variety of cell types. There are now 14 known collagens, which have numerous structural variations. The epitopes of various collagens which bind integrins remain to be fully defined. In lymphocyte interactions the only binding convincingly shown is that of type I collagen to the VLA-3 integrin. ${ }^{11}$

Cytotactin (tenascin, a hexabrachion) is an extracellular matrix protein expressed in development and in tissue repair consisting of disulphide linked hexameric glycoprotein subunits which show homology to fibronectin.
Cytotactin binds lymphocytes; the structural and functional implications of this binding are under active study. ${ }^{18}$

In SSc, in a study conducted before the full realisation of the potential for costimulatory effects, peripheral blood monocytes from patients with diffuse SSc were shown to initiate DNA synthesis on incubation with laminin. This was taken to represent cell mediated immunity to laminin in SSc without formal proof that the mitogenic signal was transmitted by the $\mathrm{T}$ cell receptor (CD3). On reconsideration, if SSc T cells were engaged with another non-laminin epitope by the $T$ cell receptor, the data are consistent with a costimulatory role for laminin in SSc and do not necessarily indicate a direct immune response role. These earlier studies should now be re-examined in light of the potential role of $T$ cell costimulatory effects.

Clinically, immune activity relevant to fibrosis can be assessed by serial determinations of serum interleukin 2 concentrations, which have been shown to correlate positively with the progression of skin tautness in a group of 43 patients with diffuse SSc. ${ }^{19}$

\section{Endothelial cell/vascular studies}

Morphological evidence for vascular/microvascular involvement in SSc has accumulated for several decades, consisting of arterial intimal proliferation, capillary dilatation and destruction, and ultrastructural evidence for endothelial injury and detachment. The biological era of study began in 1979 with the observation that SSc serum samples (and plasma) contained an endothelial cytotoxic factor, protein in nature, about $50 \mathrm{kDa}$ molecular mass, and associated with a functional decrease in serum serpin or proteinase inhibitor levels in the same serum samples. ${ }^{20} 21$ In 1990 the endothelial cytotoxic factor was tentatively identified as a serine proteinase present in the granules of activated T lymphocytes called granzyme A in the mouse and granzyme 1 in man. ${ }^{22}$ Free granzyme 1 has been identified in SSc serum samples by ELISA, and monoclonal antibody to granzyme 1 abrogates completely the endothelial cytotoxicity of SSc serum samples. Additional features of granzyme 1 are its ability to degrade collagen type IV and its inhibition by antithrombin III. What activates T cells in SSc and why they are unusually adherent to endothelium remains for further study. Clinically useful evidence of active endothelial injury can be obtained by determining plasma factor VIII-von Willebrand factor levels as an adjunct to management and treatment. ${ }^{23}$

\section{Fibroblast/matrix studies}

In 1972 mass cultures of fibroblasts from clinically and histologically affected skin of patients with SSc were shown to secrete increased amounts of collagen proteins, a phenotypic characteristic which persisted for several population doublings in vitro. ${ }^{24}$ In situ hybridisation has confirmed that mRNA for collagen $\alpha \mathrm{l}$ (I) can be detected in some but not all fibroblastlike cells in SSc skin biopsy specimens. ${ }^{25}$ Further in vitro studies have shown that colla- 
gens I, III, and VI, fibronectin, and proteoglycan are all overexpressed at the transcriptional or message level. What types of cytokines are known to coordinately upregulate these matrix molecules at the mRNA level? The leading candidate is transforming growth factor $\beta$, which, through receptors yet to be cloned and signal transduction mechanisms still poorly understood, increases mRNA levels of these and perhaps other matrix constituents. Whether this cytokine functions by increasing mRNA synthesis or decreasing mRNA decay, or both, is unclear. Direct evidence for transforming growth factor $\beta$ involvement in SSc includes the in situ identification of the cytokine in the microenvironment of fibroblasts expressing collagen mRNA in SSc skin biopsy specimens. ${ }^{25}$

It is now appropriate to determine if common protein activators of gene expression are responsible for the several matrix genes overexpressed in SSc. If one or more transacting DNA binding regulators of gene expression can be identified it might become feasible to treat SSc with diffusible oligonucleotides which prevent the expression of these regulating proteins and thereby prevent fibrosis.

Systemic sclerosis fibroblasts also exhibit a cell growth abnormality not yet linked to matrix secretion. In contrast with healthy adult human skin fibroblasts, SSc fibroblasts are resistant to the induction of quiescence by serum deprivation, continuing to synthesise DNA and to express the immediate response proto-oncogene, c-myc. ${ }^{26}$ Systemic sclerosis fibroblasts also show heightened expression of platelet derived growth factor $\alpha$ (PDGF- $\alpha$ ) receptors after eight hours' exposure to transforming growth factor $\beta$ compared with healthy fibroblasts. We propose that the PDGF- $\alpha$ ligand $/ \alpha$ receptor system affects wound healing and fibrosis. We are presently engaged in studying the regulation of expression of the $\alpha$ receptor, which is the only receptor through which PDGF- $\alpha$ induces signal transduction, whereas PDGF- $\beta$, the product of the c-sis gene, binds $\alpha$ and $\beta$ receptors equally. Ultimately, we propose that control of SSc fibroblast cell proliferation may be possible through control of PDGF- $\alpha$ ligand/ $\alpha$ receptor expression. $^{27}$

Summing up, unknown stimuli induce a T cell immune response with enhanced lymphocyte adherence to endothelium and release of granzyme 1 . These are both likely to contribute to the widespread vascular and microvascular injury that interferes with vascular permeability and exposes the interstitium to one or more fibrogenic cytokines which stimulate fibroblast proliferation and matrix gene expression resulting in fibrosis. Matrix expression continues to activate $T$ cells and the cycle begins anew. Several potential sites at which this cycle can be interrupted represent potential therapeutic strategies for SSc. ${ }^{28}$
1 LeRoy E C. Systemic sclerosis (scleroderma). In: Wyngaarden J B, et al, eds. Cecil textbook of medicine. 19th ed. Philadelphia: Saunders. In press.

2 Tan E M. Antinucleolar antibodies: diagnostic markers for autoimmune diseases and probes for cellular biology. Adv
and Immunol 1989; 44: 93-151.

3 Kahaleh M B, LeRoy E C. The immune basis for human fibrotic diseases, especially scleroderma (systemic sclerosis). Clinical Aspects of Immunology 1989; 3: 19-28.

$4 \mathrm{Kahaleh}$ M B. Vascular disease in scleroderma. Endothelial-T lymphocyte-fibroblast interactions. Rheum Dis Clin North Am 1990; 16: 53-73

5 Huffstutter J E, DeLustro F A, LeRoy E C. Cellular immunity to collagen and laminin in scleroderma. Arthritis Rheum 1985; 28: 775-80.

6 Maul G G, Jimenez S A, Riggs E, Ziemnicka-Kotula D. Determination of an epitope of the diffuse systemic sclerosis marker antigen DNA topoisomerase-I: sequence similarity marker antigen DNA topoisomerase-1: sequence similarity
with retroviral p 30 gag protein suggests a possible cause for with retroviral p30 gag protein suggests a possible cause for autoimmunity in systemi

7 Hawrylko E, Spertus A, Mele C A, Oster N, Frieri M Increased interleukin 2 production in response to human type I collagen stimulation in patients with systemic sclerosis. Arthritis Rheum 1991; 34: 580-7.

8 Kahaleh M B, Yin T. Enhanced lymphocyte-endothelial cell (EC) interaction in scleroderma (SSc) [abstract]. Arthritis Rheum 1990; 33 (suppl):A129.

9 Springer T A. Adhesion receptors of the immune system. Nature 1990; 346: 425-34.

10 Matsuyama T, Yamada A, Kay J, et al. Activation of CD4 cells by fibronectin and anti-CD 3 antibody. A synergistic effect mediated by the VLA- 5 fibronectin receptor complex and anti-CD3 antibody. F Exp Med 1989; 170: 1133-48.

11 Dang N H, Torimoto Y, Schlossman S F, Morimoto C. Human CD4 helper T cell activation: functional involvement of two distinct collagen receptors, IF7 and VLA ment of two distinct collagen receptors, IF 7
integrin family. $\mathcal{E}$ ×p Med 1990; 172: 649-52.

12 Wayner E A, Garcia-Pardo A, Humphries M J, McDonald J A, Carter W G. Identification and characterization of the T lymphocyte adhesion receptor for an alternative cell attachment domain (CS-1) in plasma fibronectin. $\mathcal{F}$ Cell Biol 1989; 109: 1321-30.

13 Beck K, Hunter I, Engel J. Structure and function of laminin: anatomy of a multidomain glycoprotein. FASEB f 1990; 4: 148-60.

14 Chiquet-Ehrismann R. What distinguishes tenascin from fibronectin. FASEB F 1990; 4: 2598-604.

15 Shimizu Y, van Seventer G A, Horgan K J, Shaw S. Costimulation of proliferative responses of resting CD4+ $\mathrm{T}$ cells by the interaction of VLA-4 and VLA-5 with fibronectin by the interaction of VLA-4 and VLA-5 with fibro
or VLA-6 with laminin. $\mathcal{F}$ Immunol 1990; 145: 59-67.

16 Shimizu Y, van Seventer G A, Horgan K J, Shaw S. Regulated expression and function of three VLA (B1) Regulated expression and function of three VLA
integrin receptors on T cells. Nature 1990; 345: 250-3.

17 Hemler M E. VLE proteins in the integrin family: structures functions and their role on leukocytes. Annu Rev Immunol 1990; 8: 365-400

18 Regg C R, Chiquet-Ehrismann R, Alkan S S. Tenascin, an extracellular matrix protein, exerts immunomodulatory activities. Proc Natl Acad Sci USA 1989; 86: 7437-41

19 Kahaleh M B, LeRoy E C. Presence of interleukin 2 in scleroderma: correlation of serum level with extent of skin involvement and disease duration. Ann Intern Med 1989; 110: $446-50$.

20 Kahaleh M B, Sharer G K, LeRoy E C. Endothelial injury in scleroderma. $\mathcal{F}$ Exp Med 1979; 149: 1326-35.

21 Kahaleh M B, LeRoy E C. Endothelial injury in scleroderma. A protease mechanism. f Lab Clin Med 1983; 101: 553-60. Kahaleh M B. The molecular mechanism of endothelial cell Kahaleh M B. The molecular mechanism of endothelial cell
(EC) injury in scleroderma (SSc): identification of granzyme 1 (a product of cytolytic T cell) in SSc sera granzyme 1 (a product of cytolytic T cell) in
[abstract]. Arthritis Rheum 1990; 33 (suppl): 67.

23 Kahaleh M B, Osborn I, LeRoy E C. Increased factor Kahaleh M B, Osborn I, LeRoy E C. Increased factor
VIII/von Willebrand factor antigen and von Willebrand factor activity in scleroderma and Raynaud's phenomenon. Ann Intern Med 1981; 94: 482-4.

24 LeRoy E C. Connective tissue synthesis by scleroderma skin fibroblasts in cell culture. $\mathcal{F}$ Exp Med 1972; 135: 1351-62.

25 Kulozik M, Hogg A, Lankat-Buttgereit B, Krieg T. Co-localization of transforming growth factor $\beta 2$ with $\alpha 1$ (I) procollagen mRNA in tissue sections of patients with systemic sclerosis. $\mathcal{F}$ Clin Invest 1990; 86: 917-22.

26 Trojanowska M; Wu L, LeRoy E C. Elevated expression of c-myc protooncogene in scleroderma fibroblasts. Oncogene 1988; 3: 477-81.

27 Ishikawa O, LeRoy E C, Trojanowska M. Mitogenic effects of transforming growth factor $\beta 1$ on human fibroblasts of transforming growth factor $\beta 1$ on human fibroblasts
involves the induction of platelet-derived growth factor $\alpha$ involves the induction of platelet-derived
receptors. $\mathcal{F}$ Cell Physiol 1990; 145: 181-6.

28 LeRoy E C, Smith E A, Kahaleh M B, Trojanowska M, Silver R M. A strategy for scleroderma (systemic sclerosis): Silver R M. A strategy for scleroderma (systemic sclerosis):
is transforming growth factor beta the answer? Arthritis Rheum 1989; 32: 817-25.
R 